\title{
DIGITAL PHOTOGRAMMETRY AS A RESOURCE FOR CUBAN CULTURAL HERITAGE: EDUCATIONAL EXPERIENCES AND COMMUNITY ENGAGEMENT WITHIN THE INNOVA CUBA PROJECT
}

\author{
G. Tucci ${ }^{1, *}$, A. Conti ${ }^{1}$, L. Fiorini ${ }^{1}$, F. Mei ${ }^{2}$, E. I. Parisi ${ }^{1}$ \\ ${ }^{1}$ Dept. of Civil and Environmental Engineering, University of Florence, Via di S. Marta 3, Florence, Italy \\ ${ }^{2}$ ARCS - Culture Solidali, Via dei Monti di Pietralata 16, Rome, Italy \\ (grazia.tucci@unifi.it, arch.a.conti@gmail.com, lidia.fiorini@gmail.com, mei@arci.it, ericaisabella.parisi@unifi.it)
}

Commission V, WG V/1

KEY WORDS: Photogrammetry, Integrated survey, Cultural Heritage, Crowdsourcing, Citizen science

\begin{abstract}
:
The Laboratory of Geomatics for Environment and Conservation of Cultural Heritage (GECO - University of Florence) has been involved since 2011 in a process of technological transfer in collaboration with the Oficina del Historiador de La Ciudad de La Habana $(\mathrm{OHcH})$. The know-how transfer concerning geomatics techniques for documentation and surveying of cultural heritage is of fundamental interest for the principal Institution designated to the protection of Havana historical centre. The first project (20112014) was mainly focused on the formation of specialized technicians from $\mathrm{OHcH}$ in the use of Terrestrial Laser Scanner (TLS), for documentation purposes of monumental and architectonical heritage. The ongoing project, INNOVA CUBA - "International and intersectoral intervention for the safeguard of the cultural heritage of the country" started in 2017, is funded by AICS - Italian Agency for Development Cooperation and coordinated by ARCS - Culture Solidali.

The introduction of digital close-range photogrammetry represents the natural progression of this educational process, thus implementing the range of innovative surveying technologies available, in terms of instruments, software and technical know-how. The two-weeks training experience in Havana (2018) provided the participants, technical staff from $\mathrm{OHcH}$ and other institutions, all the necessary knowledge to be autonomously able to choose the suitable surveying technique, depending on object characteristics and on available devices, to correctly plan the survey and evaluate its metric quality. A final workshop, for a wider audience, was the opportunity to introduce future crowdsourcing photogrammetric activities, to improve the sensitivity and engagement of local community towards heritage protection and valorisation processes.
\end{abstract}

\section{INTRODUCTION}

\subsection{The Innova Cuba Project}

The project "INNOVA CUBA - International and intersectoral intervention for the safeguard of the cultural heritage of the country" started in July 2017, with the aim to promote the use of technological and methodological innovations in the conservation and protection of cultural heritage, thus strengthening the touristic offer and the economical and social growth of the Cuban territory, through the involvement of different sectors and actors with this common goal ${ }^{1}$. The project is funded by AICS - Italian Agency for Development Cooperation and coordinated by the ONG ARCS Culture Solidali.

The role of Cuban cultural heritage is of primary importance for the human, social and economic development of the country. Both local institutions and international organizations recognize this key role and promote intervention activities in the country. The principal Institution designated to the management of Havana historical centre (La Habana Vieja), inserted in 1982 in the UNESCO World Heritage List, is the Oficina del Historiador de la Ciudad de La Habana $(\mathrm{OHcH})$. This public office was founded in 1938, with the aim of monitoring, spreading and promoting the cultural and historical values of the city. From 1967, Dr. Eusebio Leal Spengler, has started a

\footnotetext{
* Corresponding author

1 More information about Innova Cuba Project on ARCS Culture Solidali website: www.arcsculturesolidali.org
}

huge and ongoing work of reconstruction and restoration of the historical centre.

Despite the considerable efforts made in the last decades, the complete recovery of the overall heritage of La Habana Vieja is faraway, as much as the diffusion of other experiences similar to the $\mathrm{OHcH}$ in other parts of the country, which is still embryonal (e.g. the Oficina del Historiador de la ciudad de Camaguey). Furthermore, other problems concern: the substantial time required for the conservation projects, the lack of qualified technical personnel, the slow growth and diffusion of new nongovernmental management facilities in the restoration field and the subsequent competences transfer from the $\mathrm{OHcH}$ to the private sector, the scarce development of suitable ways for an efficient exploitation of the growing cultural tourism demand. The interventions of the project aspire to increase the collaboration between public institutions and private actors, along with local community to strengthen the conservation, valorisation and diffusion of the Cuban cultural heritage. The cooperation with international partners within the project, which are excellence or leader organizations in their respective field of intervention, can help the $\mathrm{OHcH}$ with technological transfer and support for the acquisition of good practices to keep carrying out its role of technical and socio-cultural innovator. Among the partners, Assorestauro is designated to training activities in the field of restoration and to the creation of a training-study Centre of restorers "REDI - Educational Centre for Restoration and Design", the Academy of Fine Art of Varsavia has the task to train the restorers to the use of a specific conservation techniques, i.e. laser cleaning, the 
University of Florence is involved with the participation of two Departments, DIDA - Department of Architecture, for the management of conservation projects and DICEA - Department of Civil and Environmental Engineering, for the transfer of surveying techniques with the use of digital photogrammetry, AITR - Italian Association for Responsible Tourism, for the development of a reference point for a conscious cultureoriented tourism, ARCI Toscana for the management and the support of new cooperatives in the restoration field.

The specific aims of the project can be summarized as follows:

i. Strengthen local capacity in the use of innovative technologies and methodologies to expand the services in favour of conservation and safeguard of cultural heritage, thus creating new professional skills;

ii. Improve the process of sensibilization, awareness and inclusion of the population in the safeguarding and enhancing of their heritage;

iii. Increase local capacity in the transfer to new nongovernmental actors, of responsibilities and tasks of the process of renovation, conservation and valorisation of historical heritage;

iv. Support the network of Heritage Cities to spread the application of new technologies linked to restoration, thus favouring the transfer of knowledge and good practices acquired by the $\mathrm{OHcH}$.

The activities expected from the participation of the GECO Geomatics for Environment and Conservation of Cultural Heritage Laboratory of DICEA, University of Florence, to fulfil each goal described above are listed below:

i. Educational activities to "train the future trainers" in the use of innovative surveying methodologies with the most up-todate Structure from Motion (SfM) close-range digital photogrammetry techniques; support in the creation of an informatic laboratory in Havana, equipped with the suitable hardware and software for data processing and the production of useful outputs for restorers, architects, engineers, urbanists, content producers for museum and touristic activities;

ii. Organization of collaborative photogrammetry events, where the already trained professionals become trainers in turn for a wider audience, under the supervision of the GECO Lab, which provides also guidelines for the basics of the photogrammetric workflow;

iii. Collaboration with non-governmental entities, such as cooperatives or professionals self-employed, to realize an autonomous laboratory for surveying and diagnostics;

iv. Diffusion of the technological transfer and support also in other provinces of the territory, with the training of technicians and specialists coming from the city of Camaguey, the third city of Cuba for extension, and the setup of an operative laboratory for documentation and conservation of cultural heritage.

\subsection{Previous experience and the role of crowdsourcing photogrammetry}

The GECO Lab has been already involved in a similar project in 2011-2014, "Salvaguarda de los valores patrimoniales y culturales que atesora el Centro Histórico de Ciudad de La Habana", concerning the technological transfer for surveying and documentation of cultural heritage. That previous project was mainly focused on the use of Terrestrial Laser Scanner (TLS) for documentation of the built and monumental heritage, by training the personnel of the Diagnostic office of the Dirección General de Projectos de Arquitectura y Urbanismo of the $\mathrm{OHcH}$. The new project, Innova Cuba, is oriented instead to expand the range of innovative surveying methodologies through the use of the most up-to-date SfM photogrammetric techniques.

Photogrammetry is a technique used since long time in cartography but with the introduction of digital photography and the advancement in information technology, it is spreading in a lot of different sectors (Fassi et al., 2017; Tucci et al., 2017). Laser scanner and photogrammetry are complementary techniques, with their specific applications and limitations. In some cases, one can result easier and more effective to use, but it usually depends on the availability of the instruments and on the final aim of the project. The main advantage in using digital photogrammetry is that it requires only a camera as acquisition device, thus being more appropriate for an extensive and flexible utilization, compared to the more expensive and bulkier laser scanner. Furthermore, it is easier to deal with photography as the starting concept for a wider audience, since it is a diffuse communicative medium, utilized and understood by many people, while the photogrammetric processing phase should be carried out by expert professionals. For this reason, only a brief training on the most suitable ways to acquire pictures useful for a photogrammetric elaboration, would be enough to involve an audience of non-expert, as professional or amateur photographers, architects, art and local history scholars and students, in the practical and first-person collection of useful data for built heritage documentation and preservation.

Such a collaborative approach in photogrammetry is becoming more and more popular in the last years, as proved by the diffusion of several projects based on crowdsourcing experiences. Citizens are identified as sensors (Goodchild, 2007) able to acquire worldwide geospatial information to share on specific websites (Heipke, 2010; Brovelli et al., 2016). One of the most famous application is the OpenStreetMap project (Neis et al., 2012) where Volunteered Geographic Information are collected by users in a web-based database, with spatial data such as roads, buildings, land use areas, or points of interest (2). Collaborative mapping, from the sociological perspective, has an intrinsic dualism, since it links social research to action and advance both science and practice (Chiesi et al., 2015), thus taking social science and research closer to society. The benefits of social engagement concerns also the academic world, usually afraid to lose its referential theorical position by practicing with community, even though action research has demonstrated the capacity to produce advances in theory and in methodology (Gustavsen, 2008; Friedman et al., 2009).

The application of crowdsourcing and citizen science to heritage documentation is a powerful source of data: the democratization of photogrammetric technology is already within everyone's means, both thanks to the rapid evolution of mobile devices, able to acquire high-resolution pictures, and to the work made in past years to develop strong and efficient algorithms for automatic data processing. The image-based 3D digitization of cultural heritage through collaborative photogrammetry has already been applied in some projects: the Project Mosul (3), for the digital reconstruction of lost Heritage (Vincent, 2015), 4D_CH World, funded in the VII European Framework with the aim of data collection over heterogeneous unstructured web resources for digital reconstruction and implementation of the Europeana (4) database (Ioannides et al., 2013), Heritage Together project (5), which produce digital 3D models of Neolithic and Bronze age remaining for

\footnotetext{
2 OpenStreeMap website: www.openstreetmap.org

${ }^{3}$ Project Mosul (REKREI) website: www.projectmosul.org

${ }^{4}$ Europeana website: www.europeana.eu

${ }^{5}$ Heritage Together website: www.heritagetogether.org
} 
crowdsourced archaeology (Griffiths et al., 2015). Other collaborative-based projects employ the acquisition of pictures from users or floating on the web to produce digital information or 3D models in a second step. The current trend is exploiting the use of mobile- and web-GIS technologies for the development of end-to-end applications, as in the REPLICATE project (Nocerino et al., 2017) and the SOCH system (Dhonju et al., 2018).

The above-mentioned democratization effect entails however the risk of lack of quality and metric control over the resulting outputs. Since this trend cannot be opposed, the best way is to use education to train professionals in a more aware use of geomatics technologies, by "training the trainers", but opening also to a wider public of non-experts, to inform them about the existence of these technologies and start training them in their correct use, with benefits also under the social aspect.

\subsection{Crowdsourced photogrammetry within the Innova Cuba project}

One of the aims expected by the project is the organization of collaborative photogrammetry events for documentation and conservation aims, where the already trained professionals will become trainers in turn. During these participative events, open to a wide public of non-experts, specifically prepared guidelines are proposed and distributed, to give some basic concepts about the preliminary planning methodologies, acquisition, processing and sharing of the outputs of a photogrammetric project for built heritage. In particular, the participants are involved in the practical aspect of the images acquisition by using their own professional or low-cost devices (reflex cameras, compact cameras, tablet, smartphones). The acquired pictures are collected in a suitable database to be later processed by the experts with dedicated software. The produced 3D models will then be used for conservative purposes (documentation and monitoring of the conservation state of the buildings) or for the creation of interactive and thematic maps for tourism valorisation. The participants to the collaborative event will finally see the model they have contributed to produce applied to the documentation, safeguard and conservation of their own cultural heritage. This and other benefits of crowdsourcing events can be listed as follows:

- information: the active involvement of the population, which directly interact and participate to the processes of safeguard and valorisation of cultural heritage, favour the appreciation and diffuse the knowledge of the national and local history, thus creating awareness and collective consciousness of being heirs and witnesses of their own territory. The training of non-expert people on new technologies, through the practical and playful acquisition of skills, can activate spontaneous processes of technological transfer to other sectors of education and professional activities;

-innovation: the attention to the historical and architectural heritage does not only mean to look at the past. Using new tools and technologies to create new experiences involving the heritage highlight the actuality of the past, to help the community understanding the changes to guide future decisions for coming generations, fostering tourism, investment and employment. These collaborative initiatives also accelerate and ease the process of data collection but reserving to the specialized operators the work phases which require more in-depth skills, as data processing;

- identification: the individual contribution to obtain useful results for the community increase the sense of social integration, the personal wellness and life quality. Furthermore, these activities favour the chance to deepen interpersonal relationships, interactions, and bonds inside the local community. This sense of engagement can become the engine of training and work to stimulate actions towards shared goals.

\section{EDUCATIONAL ACTIVITIES IN HAVANA}

The educational activities were carried out during the first mission of the GECO Lab in Cuba, from January $20^{\text {th }}$ to February $4^{\text {th }}, 2018$. The two-weeks tutorial has involved twelve participants, with different backgrounds in the field of engineering, architecture, anthropology, photography, and others. Most of the students were already active as operators in Cuban institutions for cultural heritage safeguard, as in the Oficina del Historiador de La Habana and de Camaguey, Plan Maestro and Empresa Restaura.

The principal goal of the first mission has been to give the participants the cognitive instruments for being able to autonomously choose the suitable surveying techniques depending on i) the characteristics of the object and ii) the available instruments, iii) to correctly plan the acquisitions, iv) to evaluate the metric quality of the project.

The GECO Lab also followed, from Italy, the setup of two designated laboratories for data collection and processing, one placed in the offices of the Departamento Diagnóstico $y$ Levantamiento - Empresa de Proyectos de Arquitectura y Urbanismo Restaura (an autonomous enterprise which works as a spin-off of the $\mathrm{OHcH}$ ) in Havana and another one in Camaguey. The purchase of each hardware and software was carefully evaluated to give the best up-to-date instruments to be fully operative in digital surveying: 4 workstations, 1 printers, 3 reflex full-frame cameras Nikon D750, 5 reflex APS-C cameras Nikon D3300 equipped with AF-P 18-55 mm objectives, 3 Nikkor objectives for each focal length of $50 \mathrm{~mm} \mathrm{f} / 1.4 \mathrm{G}, 24$ $\mathrm{mm} \mathrm{f} / 1.8 \mathrm{G}$ and $105 \mathrm{~mm} \mathrm{f} / 1.4 \mathrm{E}, 3$ tripods, 1 total station, licences for cloud points and images processing, 3DF Zephyr Aerial and other open-source software. All the in-class training activities were carried out in the specifically prepared laboratory in Havana, also to test all the instruments supplied by the project. Furthermore, offline procedures and licences were provided to overcome the absence of internet connection.

The two-weeks course activities were organized in the following steps:

- Theoretical lectures

- Surveying activities on the field

- Data processing

- Final workshop

All the listed activities were held in La Habana Vieja neighbourhood, as showed in Fig. 1. The participants were divided into three groups in order to use one of the three workstations each.

\subsection{Theoretical activities}

The didactic method adopted for the education activities has been the object-based approach (Hess et al., 2017). The "learning-by-doing" didactic method is proved to be more effective compared to traditional frontal lessons (Kravchenko et al., 2016), by carrying out specific projects about local heritage. Practical activities are also followed by short lessons explaining some theorical concepts, with the support of multimedia materials, such as videos, tutorials, presentations and lectures notes about useful topics (Kosmatin Fras et al., 2016). The lessons covered general topics, as methods for integrated surveys and some experiences of the GECO Lab, but focusing 
on key aspects for photogrammetry, as elements of photography, the correct planning of acquisitions, the geometry of picture acquisition, the object scale, the Ground Sample Distance (GSD) concept and the graphicism error theory (Fig. 2).

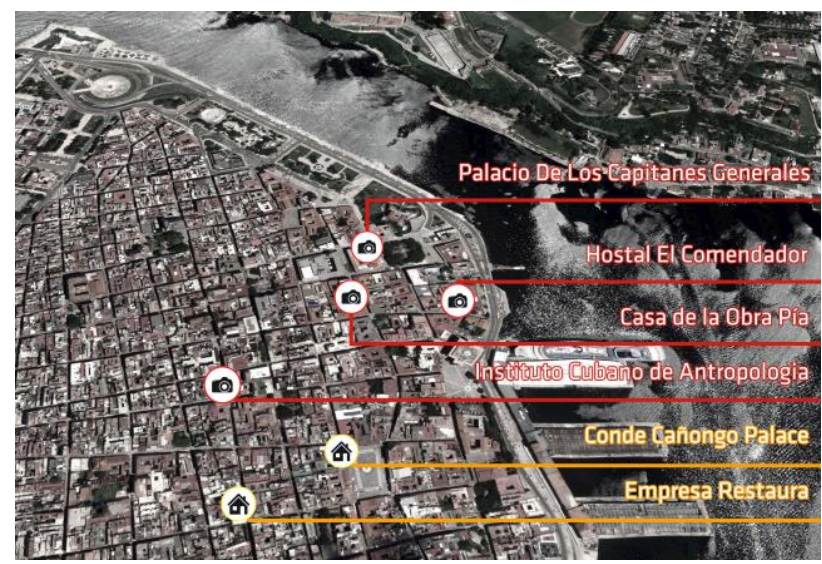

Figure 1. Map of the activities carried out in La Habana Vieja: red stands for photogrammetric acquisitions, yellow for the inclass activities and final seminar locations

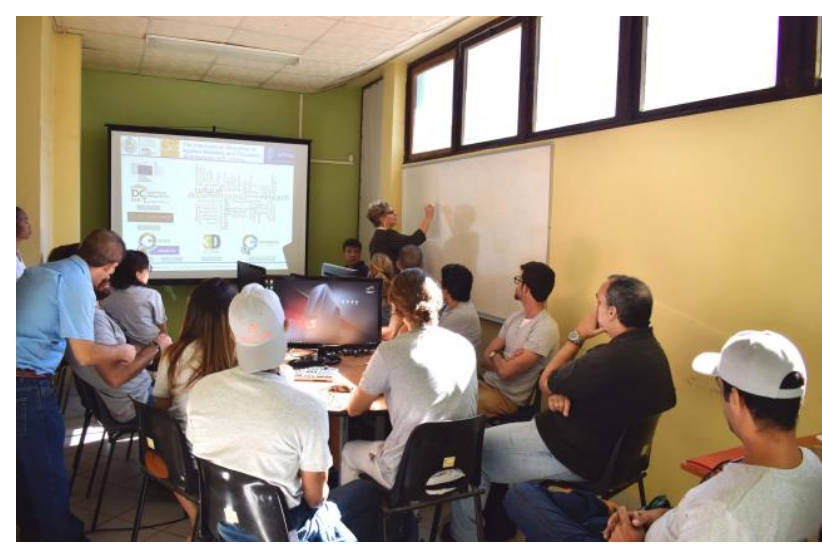

Figure 2. Theoretical in-class activities at the Departamento Diagnóstico y Levantamiento - Empresa de Proyectos de Arquitectura y Urbanismo Restaura

A significant problem has been represented by the absent or the difficult internet connection, thus forcing the preparation of the entire didactic material before leaving Italy and by previously downloading every possible source of information. From the didactic point of view, nowadays seems very anachronistic teaching without the chance to search for or download supporting material from the internet; by the students' side it represents the impossibility to autonomously integrate the own knowledge with multimedia supports (e.g. video tutorials for the use of software or blog for problems solutions). The provided software with offline licences can be affected, with time, by the lack of internet connection as well, for example in the case of updates. For this reason, one further goal of the Cuban partners is to provide internet connection to the laboratory as soon as possible.

Some technical lessons were dedicated to the introduction of the software chosen in this project for the photogrammetric workflow, 3DF Zephyr Aerial (6) by 3DFlow s.r.l., an Italian company. Three licences were provided, one for each workstation and further details about the interface were given during practical activities.

One recurring issue that emerged during the surveying activities for data acquisition and during data processing was the need to learn some basic information on topography by the students. Most of them had never studied or practiced topography, others as autodidacts or knew it only superficially. For this reason, an out of schedule basic lesson about topography was given, highlighting the importance of this techniques to determine the correct reference system as the fundamental base for integrated surveys. An indoor exercise on topographical networks, has been also arranged to help the students understanding also the practical application of the given notions.

\subsection{Surveying activities}

During the organization of the first mission of the project it has been requested to Cuban partners some advices on the possible built heritage to be surveyed as practical activities during the tutorial. Empresa Restaura has proposed some themes of interest around La Habana Vieja, of which two buildings were chosen for integrated surveys and to test the photogrammetric approach on the architectonical scale (see Fig. 1):

- the portal of the Casa de la Obra Pía

- the façades of the Hostal El Comendador

The first selected case was the high baroque portal of the Casa de la Obra Pía, placed in a very narrow street compared to the height of the buildings along. The students had the opportunity to test different devices to acquire the pictures, from professional reflex cameras with a full-frame sensor, to entrylevel reflex cameras with APS-C sensor, and low-cost devices as smartphones, to compare the different quality of the data and results obtained. The pictures were acquired also from the first floor of the facing building to acquire the entire height of the portal. The final aim was to compare different operative processes and experience the pros and cons for each one. The model was scaled by using specifically made scale bars and oriented by materializing a reference system directly on the building façade (Fig. 3A).

The second case study interested the facades of the ancient house of Pedro Regalado Pedroso y Zayas, where today is placed the Hostal El Comendador. This building was selected to allow the participants conducting a complete experience of an integrated survey applied to a building of reduced dimensions. The students practiced with a total station to acquire the coordinates of the targets used as control and check points for close range photogrammetry, and the targets used to align and roto-translate the scans acquired by TLS. The aim of this acquisition was to show the possibility of using the data in a complementary and integrated way within a restoration or documentation project, by orienting the cloud points obtained by different technologies in a common reference system, given by topography (Fig. 3B, 3E).

The last cases study have focused on two small objects, to give a complete overview of the potentialities of photogrammetry on a multi-scale level. The Instituto Cubano de Antropología made available some precious pre-colombian objects as tests: a small feminine figure and a head made of stone. The specific aim was to examine the appropriate acquisition modalities for such small archaeological artifacts and the different approach depending on the final goal: to obtain accurate and complete models with high resolution for archiving or study needs or with different level of decimation to use them in virtual museums or for online visualization (Fig. 3C).

\footnotetext{
${ }^{6}$ Software information on 3DFlow website: www.3dflow.net
} 


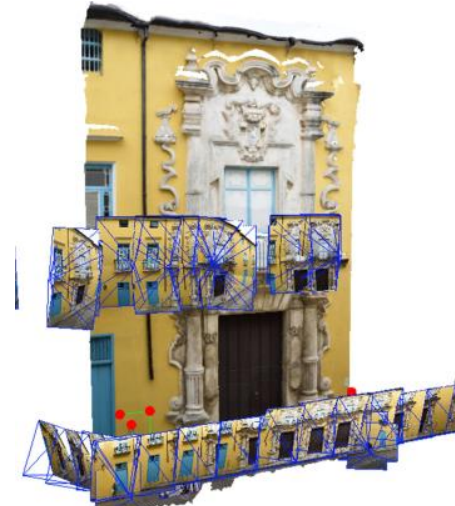

Fig. 3A

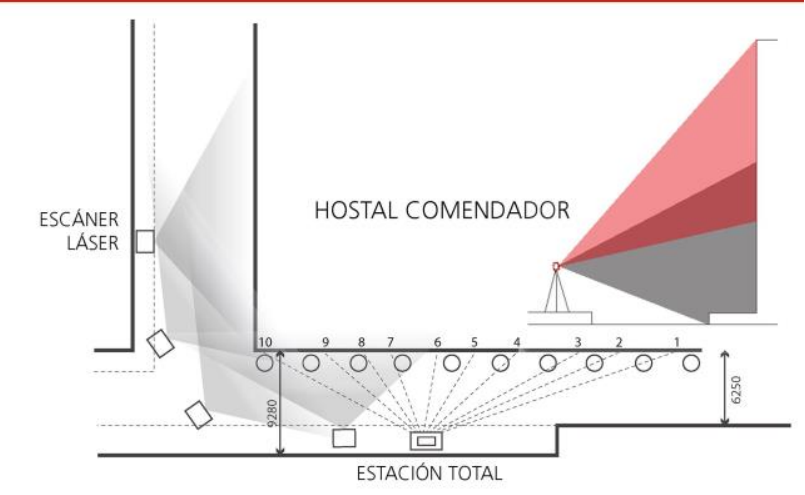

Fig. 3B
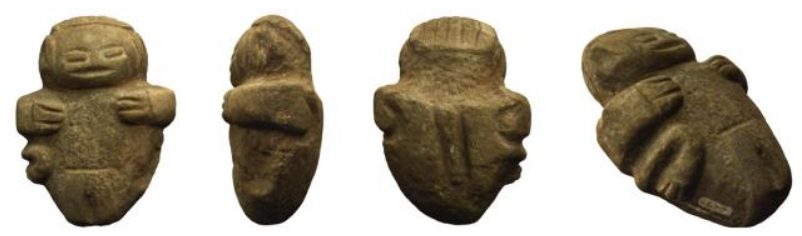

\section{Fig. 3C}

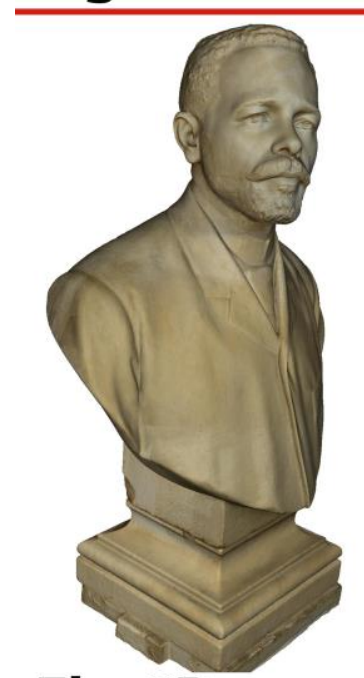

Fig. 3D

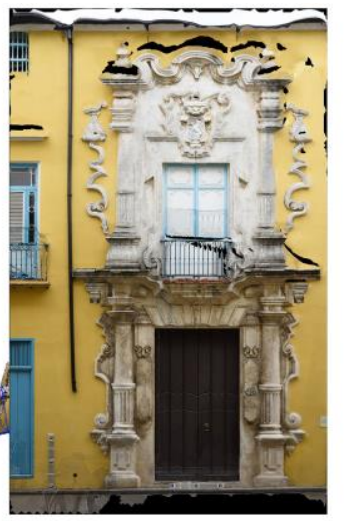

\section{.}

.

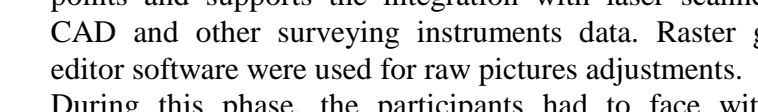

During this phase, the participants had to face with some common difficulties in acquiring pictures for a photogrammetric project on the architectonical scale, as reduced section of the street, scarce flexibility in acquiring the images from an elevated point of view, impossibility to distribute the control point all over the façade, presence of trees and other obstacles. As a result, they had the chance to test the critical issues that can occur in the processing phase, such as different resolution in the bottom part compared to the upper part of the façade, holes in correspondence with cornices or protrusions, artefacts along thin elements as gratings, distortions gathered around the marginal or distant areas from the control points of the model.

The processing of data coming from different sources and technologies, as TLS, photogrammetry and topography, has been also the occasion to recall the concept of reference system (global and local) and the use of User Coordinate System (UCS) in different software (CAD, laser scanner data management.). The final goal of both the architectural scale projects has been the production of a 3D surface model, metrically validated and conveniently oriented, and the respective orthophotos.

For what concern the reduced scale, instead, the main final consideration was that having a proper set, with the correct illumination, position and stability of the object, is fundamental in indoor photogrammetric projects of small objects. One representative student coming from the Instituto Cubano de Antropología was designated to learn how setting-up such a controlled space, devoted to the acquisition of the museal collections, in a continuous and productive way.

\subsection{Further activities}

The first mission has concluded with a final seminar at the Conde Cañongo Palace, where the participants presented the results of the two-weeks training to a wider audience, represented by operators, specialized technicians and students from Havana leader Intitutions in the conservation of cultural heritage, as the $\mathrm{OHcH}$, the Colegio Universitario San Gerónimo and the Escuela Taller Gaspar Melchor de Jovellanos (Fig. 4). The tutors presented an overview of the digital technologies for the documentation, conservation and valorisation of Cuban cultural heritage and a project for collaborative photogrammetry events. In fact, this workshop was a fruitful opportunity to get in contact with potential interested targets for other educational or collaborative initiatives. At the same time, the final seminar, was also an occasion for the tutors to evaluate the work done by the students, whom followed with considerable attention and enthusiasm the teachings they have learned during the tutorial. 
The days of the course were also the occasion to see and optimize previously performed projects and support the participants who already had experiences in digital surveying. The participants have extensively taken advantages of this opportunity for discussion, to show the tutors some critical issues they have found in digital surveys already carried out and to solve doubts and questions. An example is the case of the equestrian monument of Calixto García, obtained by a previous TLS survey carried out by Empresa Restaura. The model was very difficult to handle due to the huge number of points. Opensource software, ISTI-CNR MeshLab and CloudCompare were used to show some reality-based modelling techniques, as decimation and segmentation of the model, to produce a mesh with ambient occlusion shading, and to reduce the overall size of the model.

Another proposed case of interest concerned the digitization project of the entire collection of busts of the most significative historical national personalities, at the City Museum in the Palacio De Los Capitanes Generales. The photogrammetric project and the acquisition of the pictures of one sample bust (Antonio Maceo) were carried out from the tutors of the GECO $\mathrm{Lab}$, and then processed independently in the respective Countries by the participants and by the tutors, to compare the different workflows and results (Fig. 3D). The final aim was the physical reproduction of the models by $3 \mathrm{D}$ printing.

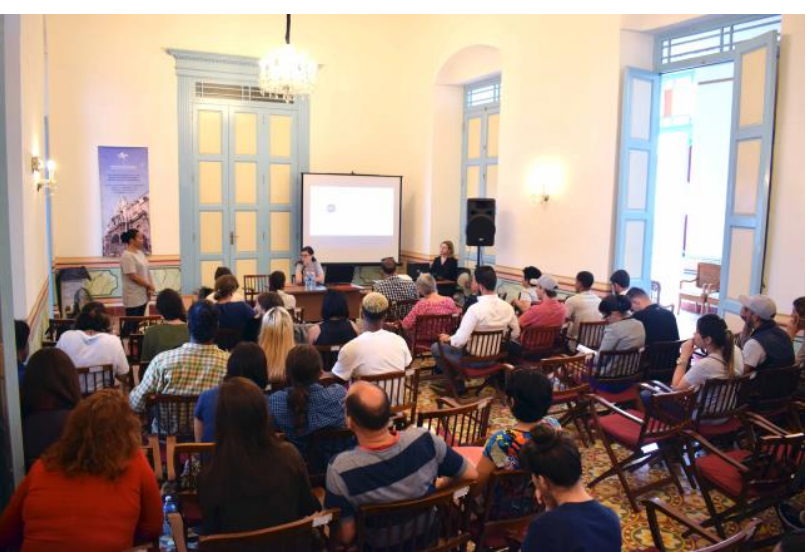

Figure 4. The final seminar at the Conde Cañongo Palace

\section{FIRST RESULTS, ONGOING ACTIVITIES AND FUTURE PERSPECTIVES}

\subsection{First results of the Innova Cuba project}

Some evaluations over the activities carried out by the GECO Lab during the first mission within the Innova Cuba operation framework can be done by recalling the objectives stated in section 1.1:

i. The educational activities to "train the future trainers" conducted during the two-weeks tutorial represents a successful experience for both the tutors, satisfied of the results presented during the final seminar, and the participants, which showed a sincerely interest and an active participation to all the phases. The participants were finally able to handle a photogrammetric workflow from the planning of the project up to the production of the final outputs (3D models and orthophotos). Although photogrammetry was the main technological-transfer focus of this first mission, the students were trained also on the use of integrated techniques, as TLS and topography, to have a complete overview of the available surveying methods and instruments. Furthermore, the final outputs produced fulfilled the requirements of usefulness for different aims: orthophotos for restoration purposes (material decay and degradation mapping), high-resolution 3D models for structural monitoring and restitution of plans and sections, and low-resolution 3D models for web- or mobile-based visualization for tourism purposes. Another goal was achieved with the installation of an equipped laboratory for digital surveying and data processing in Havana: the hardware specifics of the workstations showed adequate performances for data processing and the software worked offline as expected. However, the tutors gave their complete willingness to remotely carry on the supporting activities of the laboratories, in order to maintain the created connections and to transform the in-situ training in distance learning supervision;

ii. On 27-28 June 2018 the first test event of collaborative photogrammetry took place in Havana, organized by the Cuban partners. The target of the workshop were the students of the fourth year of the CUJAE - Universidad Tecnológica de La Habana (15 people). The event was organized in two days: the first day was dedicated to explaining theoretical basic concepts, such as elements of photography, basics of photogrammetric surveying, software tutorial and the theme of collaborative photogrammetry, by recalling the structure of the course. The second day was dedicated to pictures acquisition on the field and data processing. The participant students gathered also academic credits, as a further incentive for participation and also fulfilling other requirements of the project, i.e. the curricular update of the courses of study. The workshop was entirely handled by four participants to the first training, whom became trainers in turn. This first experience represented an applicative test of the two different aims of this project, as highlighted in section 1.2: on one side an indepth training for specialized technicians who have to deal with conservation issues and will be future trainers in turn, on the other the transfer of basic concepts about photogrammetry (by focusing on the acquisition phase) to a wider audience in little time;

iii. The involvement of Empresa Restaura and of its participant to the course, represents an example of collaboration with a spin-off of the $\mathrm{OHcH}$. The work done with them was very productive under several points of view: the diligence of their members in following the course, the interest showed for the treated topics, the several questions and requests done, as well as the demand of supervision of their ongoing works also at distance (photogrammetric acquisitions of the national heroes' busts at the City Museum). The cognitive transfer wasn't only unidirectional, but it was a mutual interchange from both sides: of knowledge, skills and methodologies for students and of better communication and teaching methods for the tutors. In particular, the questions and doubts posed during the course were useful indicators of which topics to deepen or integrate during the classes;

iv. The extension of the technological transfer to other qualified personnel in addition to the one of Havana, has focused on another Heritage City, Camaguey. Two technicians from there were trained during the course and the suitable hardware and software were provided to set-up a laboratory for digital surveying activities and data processing. The tutors gave the maximum availability to them too, in order to support and supervise their first work independent activities (at least three) which has to be performed to fulfil the project requirements. 
Another signal of appreciation of this first experience is the request, after the final seminar of the course, from other institutions representatives, of information about the possibility of receiving similar training and set-up their own laboratory. The interest showed proved the success of the first educational activities and the possibilities to further spread them towards other educational institutions, also to improve the academic courses offer.

Furthermore, an anonymous assessment questionnaire was submitted to the twelve participants few weeks after the end of the course. The final feedbacks can be summarized by following the six posed questions:

- was the previous background enough to follow the course?

The previous background of almost all the participants allowed them to follow the course topics, although most of them had not specific competences on the geomatics field. Others had superficial experience in the use of TLS or software for 3D modelling for architectural planning, 2D CAD or graphic software.

- was the teaching method of the tutors appropriate? The student defined as excellent the teaching methodology and the ability of the tutors to transfer their knowledge to them. They also expressed satisfaction for the logic organization of the topics also by considering the few days available, the addition of themes on-request, developed with great professionalism, patience and quality.

- was the duration of the course appropriate to the treated topics? The time dedicated to the course is the only critic feedback. Almost all the participant expressed their will to have more time for practical surveys and to properly face the most complex arguments regarding the topics of the course. Although it was logistically impossible to dedicate more time to the course, the project also includes other two missions: another one in Havana for the GECO tutors and one in Italy for cuban participants.

- is there an aspect of the course to deepen in the second part (topography, laser scanner use, knowledge of software) or themes already covered in the first part to be repeated? In this case each participant expressed its own interest in specific themes related to the field of work. The principal topic they asked for supplementary in-depth analysis is topography. This is also because the tutors underlined with emphasis the importance of this technology for integrated surveys. Other requests concerned more information and practical activities with TLS (which was the main topic of the previous project although the participants were not the same) to integrate all the innovative surveying technologies learned, as far as more information on 3D modelling.

- was the organizational/logistical aspect suitable to the course activities? There was no complaining about the organizational and logistical aspect.

- how is it going on the work started within the course? Not all the students had the chance to continue the work after the course, for the lack of instruments or adequate space. Some of them are gearing up for the suitable equipment and others have already started to transfer the acquired knowledge to their colleagues and during the collaborative events. Some practical applications of photogrammetry were made for the acquisition of the busts, with the aim of physical reproduction and of facades during the collaborative event for conservation purposes.

\subsection{Future perspectives}

Among the future appointments of the project Innova Cuba, two further missions are planned: another mission for the GECO
Lab to Camaguey and Havana and an in-depth training course in Italy for some of the participants to the first course. The principal aims of the next mission in Cuba will be:

- to verify, deepen and complete the photogrammetry skills of the participants also by considering the autonomous activities carried out meanwhile;

- to visit Camaguey in order to supervise the development of a laboratory of surveying and diagnostics, as for the one in Havana;

- to organize other crowdsourcing events of photogrammetry with a selected target of non-experts as audience and with a precise final purpose, e.g. the creation of a collaborative map of Havana, as in the OpenStreetMap project, with 3D models of the built heritage to celebrate the $500^{\text {th }}$ anniversary of the city (in 2019).

The following mission to complete the technological transfer is the visit of a group of participants to the GECO Lab in Florence. In this way, the selected people will complete their training course with personal-dedicated time to cover all the topics of interest and the single doubts. It will be also an occasion to work with the laboratory instruments and to participate to tailored or ongoing project of the lab, from the planning, to the acquisition, the processing and the production of the outputs. They can then teach and integrate the previous knowledge of the other participants as soon as they come back to Cuba, thus concluding the training process by always maintaining a connection at distance.

\section{CONCLUSIONS}

The Innova Cuba project represents the natural progression of the previous project "Salvaguarda de los valores patrimoniales y culturales que atesora el Centro Histórico de Ciudad de La Habana", to complete the technological transfer of geomatics techniques for documentation and surveying of cultural heritage to specialized technicians of Cuban institutions devoted to protection and valorisation of cultural heritage. The first mission of the GECO Lab to Cuba has been the occasion to introduce close-range digital photogrammetry to the participants with a two-weeks course, by applying this methodology to real surveys. The educational object-based approach, oriented to territorial heritage cases of study, was didactically productive, as highlighted by the participants feedbacks. The constant connection, also at distance, with the GECO Lab, as well as the following missions, should assure at the end of the project, a complete training of autonomous figures able to handle photogrammetric projects as far as integrated surveys, thank also to the provided instruments and laboratories. Another important aspect of the project involves the opening to a wider audience of photogrammetry, by the organization of collaborative events, to explore the social benefits of crowdsourcing for documentation of cultural heritage and to produce useful products for tourism enhancement.

\section{ACKNOWLEDGEMENTS}

The authors wish to thank the ARCS - Culture Solidali staff for the administrative support in the organization of the first mission and the Dirección de Cooperación de la $\mathrm{OHcH}$ for the arrangement of the final seminar and other activities in Cuba. Special thanks go to the Eng. Alberto Hernández Oroza, chief of the Departamento Diagnóstico y Levantamiento - Empresa Restaura, for his careful cooperation in all the educational 
activities and to the participants of the course for their enthusiasm.

This work has been carried out under the GAMHer project: Geomatics Data Acquisition and Management for Landscape and Built Heritage in a European Perspective, (PRIN2015 n.2015HJLS7E).

\section{REFERENCES}

Brovelli, M.A., Minghini, M., Zamboni, G., 2016. Public participation in GIS via mobile applications. In: ISPRS Journal of Photogrammetry and Remote Sensing, Vol. 114, pp. 306-315 doi.org/10.1016/j.isprsjprs.2015.04.002

Chiesi, L., Costa, P., 2015. One strategy, many purposes. A classification for cultural mapping projects, Cultural Mapping as Cultural Inquiry, Routledge, London, pp. 69-85.

Dhonju, H., Xiao, W., Mills, J., Sarhosis, V., 2018. Share Our Cultural Heritage (SOCH): Worldwide 3D Heritage Reconstruction and Visualization via Web and Mobile GIS. In: ISPRS International Journal of Geo-Information, Vol. 7(9), pp. 360

doi.org/10.3390/ijgi7090360

Fassi, F., Campanella, C., 2017. From daguerreotypes to digital automatic photogrammetry. Applications and limits for the built heritage project. In: The International Archives of the Photogrammetry, Remote Sensing and Spatial Information Sciences, Vol. XLII-5/W1, pp. 313-319,

doi.org/10.5194/isprs-archives-XLII-5-W1-313-2017

Friedman, V. J., Rogers, T., 2009. There is nothing so theoretical as good action research. In: Action Research, Vol. 7(1), pp. 31-47

doi.org/10.1177/1476750308099596

Goodchild, M.F., 2007. Citizens as sensors: the world of volunteered geography. In: GeoJournal, Vol. 69(4), pp. 211 221

doi.org/10.1007/s10708-007-9111-y

Griffiths, S., Edwards, B., Karl, R., Labrosse, F., Miles, H., Moeller, K., et al., 2015. Crowd-sourcing archaeological research: HeritageTogether digital public archaeology in practice. In: Internet Archaeol., Vol. 40 doi.org/10.11141/ia.40.7.3

Gustavsen, B., 2008. Action research, practical challenges and the formation of theory. In: Action Research, Vol. 6(4), pp. 421-437

Doi.org/10.1177/1476750308094130

Heipke, C., 2010. Crowdsourcing geospatial data. In: ISPRS Journal of Photogrammetry and Remote Sensing, Vol. 65, pp. $550-557$

doi.org/10.1016/j.isprsjprs.2010.06.005

Hess, M., Garside, D., Nelson, T., Robson, S., Weyrich, T., 2017. Object-Based Teaching and Learning for a Critical Assessment of Digital Technologies in Arts and Cultural Heritage. In: ISPRS - International Archives of the Photogrammetry, Remote Sensing and Spatial Information Sciences, Vol. XLII-2/W5, pp. 349-354

doi.org/10.5194/isprs-archives-XLII-2-W5-349-2017
Ioannides, M., Hadjiprocopis, A., Doulamis, N., Doulamis, A., Protopapadakis, E., Makantasis, K., et al., 2013. Online 4D reconstruction using multi-images. In: ISPRS Annals of Photogrammetry Remote Sens. Spat. Inf. Sci., Vol. II-5/W1(1), pp. $169-174$

doi.org/10.5194/isprsannals-II-5-W1-169-2013

Kosmatin Fras, M., Grigillo, D., 2016. Implementation of active teaching methods and emerging topics in photogrammetry and remote sensing subjects. In: The International Archives of the Photogrammetry, Remote Sensing and Spatial Information Sciences, Vol. XLI-B6, pp. 87-94.

doi.org/10.5194/isprsarchives-XLI-B6-87-201

Kravchenko, I., Luhmann, T., Shults, R. 2016. Concept and Practice of Teaching Technical University Students to Modern Technologies of 3d Data Acquisition and Processing: a Case Study of Close-Range Photogrammetry and Terrestrial Laser Scanning. In: The International Archives of the Photogrammetry, Remote Sensing and Spatial Information Sciences, Vol. XLI-B6, pp. 65-69.

doi.org/10.5194/isprsarchives-XLI-B6-65-20

Neis, P., Zipf, A., 2012. Analyzing the Contributor Activity of a Volunteered Geographic Information Project - The Case of OpenStreetMap. In: ISPRS International Journal of GeoInformation, Vol. 1, pp. 146-165 doi.org/10.3390/ijgi1020146

Nocerino, E., Lago, F., Morabito, D., Remondino, F., Porzi, L., Poiesi, F., et al., 2017. A smartphone-based 3D pipeline for the creative industry - The REPLICATE EU project. In: International Archives of the Photogrammetry, Remote Sensing and Spatial Information Sciences, Vol. XLII-2/W3, pp. 535541

doi.org/10.5194/isprs-archives-XLII-2-W3-535-2017

Tucci, G., Bonora, V., Conti, A., Fiorini, L., 2017. High-quality 3D models and their use in a cultural heritage conservation project. In: The International Archives of the Photogrammetry, Remote Sensing and Spatial Information Sciences, Vol. XLII2/W5, pp. 687-693,

doi.org/10.5194/isprs-archives-XLII-2-W5-687-2017

Vincent, M. L., Gutierrez, M. F., Coughenour, C., Manuel, V., Bendicho, L. M., Remondino, F., Fritsch, D., 2015. Crowdsourcing the 3D digital reconstructions of lost cultural heritage. In: 2015 Digital Heritage, pp. 171-172 doi.org/10.1109/DigitalHeritage.2015.7413863 